\title{
Oral dysplasia and risk of progression to cancer
}

\section{Abstracted from \\ Mehanna HM, Rattay T, Smith J, McConkey CC.}

Treatment and follow-up of oral dysplasia - a systematic review and meta-analysis. Head Neck 2009; 31: 1600-1609

Address for Correspondence: Hisham M. Mehanna, Department of Head and Neck Surgery,

Institute of Head and Neck Studies and Education, University Hospitals Coventry and Warwickshire,

Coventry, United Kingdom. E-mail: hisham.mehanna@uhcw.nhs.uk<mailto:hisham.mehanna@uhcw.nhs.uk>

\section{Questions: What is the risk of and interval to progression to oral cancer in patients diagnosed with oral dysplasia?}

Data sources Medline, EMbase and Cochrane databases

Study selection Studies were included if they reported data on patients with a histologically-confirmed diagnosis of oral dysplasia. They also had to study at least one outcome measure and one intervention method or clinical risk factor. Outcome measures included malignant transformation rate (MTR) and time to malignant transformation (TMT). In studies on oral lesions, which contained a defined subset of patients with oral dysplasia, this subset but not all patients with oral lesions were included in the meta-analysis. Observational studies were included in the review due to the scarcity of randomised controlled trials with adequate follow-up period.

Data extraction and synthesis Quality assessment was undertaken independently by two reviewers. When there was disagreement, a third reviewer was consulted. Quality criteria were agreed upon a priori, and the authors used a combination of quality assessment methods. Eligibility of studies was determined independently from the abstracts by two reviewers blinded to each other's selections. Outcome data were abstracted independently by a researcher and a statistician and checked by a third reviewer. Heterogeneity was assessed graphically in a forest plot and a meta-analysis was conducted. Subgroup analysis was performed by histologic grade, clinical risk factors and treatment modality.

Results Fourteen non-randomised studies, reporting on 992 patients, were included. There was considerable heterogeneity between studies. The mean overall MTR was $12.1 \%$ (95\% Cl: $8.1 \%, 17.9 \%)$ and the mean TMT was 4.3 years. Histologic grade significantly affected mean MTR ( $p$ 0.008). Furthermore, lesions that were not excised demonstrated considerably higher MTR than those that were excised $(p=0.003)$

Conclusions Oral dysplasia showed a significant rate of transformation to cancer, which was related to grade, and was decreased significantly but not eliminated by excision. Findings suggest the need for surgical excision and continued surveillance, particularly in high-grade lesions.

\section{Commentary}

Oral dysplasia is detected by clinicians in the form of leukoplakia, a white patch in the oral cavity that cannot be diagnosed as any other known disorder. These lesions carry an increased risk of malignant progression to oral squamous cell carcinoma. The early diagnosis, treatment and surveillance of these precancerous lesions are important predictors in preventing progression to oral cancer; therefore, this meta-analysis is very relevant.

The aim of this work was to inform an evidence-based management and surveillance policy for oral dysplastic lesions. The authors assessed the risk of and interval to progression to cancer in patients diagnosed with oral dysplasia. This is an area of clinical practice that demonstrates variability and lacks high-quality evidence.

The review had rigorous inclusion and exclusion criteria. 14 prospective and retrospective studies were identified. Data were subcategorised by histologic grade of the lesion and management strategy. The relative risks of malignant transformation for gender, lesion site, continuation of smoking and alcohol consumption after diagnosis were also extracted, where available.

Results showed that oral dysplasia carries a significant rate of transformation to cancer (12.1\%). Furthermore, surgical excision decreased the risk of transformation by more than half, but did not eliminate it. Subgroup analysis by dysplasia grade and treatment modality showed no significant differences in TMT. This may be due to the limited availability of data.

There was insufficient information in included studies to assess the effect of clinical risk factors on progression to malignancy. Nonetheless, malignant progression seemed to be associated with lesions of the tongue (relative risk $=1.87$; CI: $1.11,3.17$ ). The review showed that continuous smoking and alcohol use after diagnosis did not have any effect on malignant transformation. It is likely that this finding is due to the limited number of studies containing information on smoking and alcohol use and the reported heterogeneity between the two studies with data on smoking. Moreover, although oral dysplasia is uncommon in non-users of tobacco and alcohol, a study by Jaber ${ }^{1}$ found that oral dysplasia may develop in persons who have never used tobacco or alcohol, suggesting that risk factors other than alcohol and tobacco may exist.

The work also found that sex is a risk factor for oral dysplasia with women having a higher risk than men. Although this finding lacked statistical significance, it is in line with findings from other studies. $^{1,2}$ Lan and colleagues ${ }^{2}$ reported that risk of mild-moderate and severe dysplasia was 2.40 and 3.11 times higher, respectively, in women than men. They also reported that the malignant 